pregnant uterus. I have studied the question very closely and carefully. The torsion may occur early in the pregnancy or it may be delayed till delivery. The occurrence of acute torsion at the time of delivery (or abortion) is due to the rapid diminution of the uterus and the movement which this organ as it sinks into the pelvis imparts to the tumour. A case of very great interest in relation to this question was reported by Dr. St. John Edwards in THE LANCET of $186 \mathrm{I}^{11} \mathrm{~A}$ woman, 24 years of age, had for at least two years previously an ovarian tumour on the right side which did not attract much attention until she became pregnant in 1860. In October of that year she was delivered of a child who only survived a few days. Immediately after the birth of the child the tumour shifted over to the middle of the abdomen. On August 5 th, 1861, this woman was delivered of anotber child at the seventh month; the infant lived a few hours. After the child was born the tumour was found in the middle line of the abdomen. The patient died three days later. At the post-mortem examination the pedicle of the cyst was found $t$ wisted one and a half turns; it contained four litres of blood-stained fluid. The ovary was on the surface of the cyst, which was therefore parovarian in origin. I have often referred to this case, for it illustrates very well the fact that the majority of ovarian cysts, when they rotate axially, turn to the middle line and instead of occupying a lateral position lie in the hypogastrium. I have on nine occrsions performed ovariotomy when the tumour has been noticed a few days after labour. The patients state that they notice that the abdomen does not return to its proper size and in some instances that it seems to enlarge again. This enlargement in some of the cases was due to bleeding into the cyst. A marked feature in all the cases, and one which led to some doubt in the diagnosis, was the way in which the tumour occupied the middle line of the abdomen and simulated an enlarged uterus and in all the cases axial rotation had occurred. In some of them the skin of the abdomen exhibited the peculiar yellow pigmentation so often associated with pregnancy, but which very rapidly disappeared after the removal of the tumour. The axial rotation of an ovarian cyst not only menaces the life of the patient immediately on its occurrence, but the longer if is allowed to persist it increases the difficulty, and therefore the risk, of ovariotomy by establishing extensive athesions between the tumour and the adjacent viscera, especially the intestines.

Another danger which threatens a puerperal woman when she has an ovarian cyst is the liability of the cyst to suppurate. A critical study of a large collection of histories of patients who have had their "labour" complicated with ovarian cysts shows that in the majority of instances the cysts have twisted pedicles and that in a fair proportion of cases suppuration has occurred in the cyst. In many of the cases a ruptured cyst has been found.

It is now a well-attested fact that ovariotomy can be successfully performed even while labour is in progressthat the operation in no way interferes with the contraction of the uterus. Single and even double ovariotomy can be successfully performed in the puerperium without in any way interfering - with either the involution of the uterus or lactation. Therefore, it cannot be too strongly urged that when a puerperal wiman known to possess an ovarian tumour exhibits unfavourable symptoms ovariotomy should be resorted to without delay.

14 The LaNcet, Oct. 5th, 1861, p. 336.

Moretonhampstead Hosp tal.-The hospital which the Hon. W. F. D. Smith, M.P., has endowed with $£ 2000$ and presented to Moretonhampstead, Devonshire, as a memorial to his late father, was opened on Jan. 26th. Owing to the death of the Queen the formal opening by Lady Esther Smith was abandoned.

Newton Abвot Building By-Laws.-At Newton Abbot (Devon), on Jan. 29th, a builder was charged with $t$ wo breaches of the building by-laws of the Newton Abbot Rural Council-viz., that he varied the plans of cottages after they had been approved by the council and that he permitted the houses to be occupied without obtaining the usual "occupation certificate." The magistrates fined him £l and costs in each case and also a penalty of 18 . a day until the by-laws are complied with.

\section{A PLEA FOR THE MORE CAREFUL STUDY OF THE SYMPTOMS OF PERFORATION IN TYPHOID FEVER WITH A VIEW TO EARLY OPERATION.}

BY WILLIAM OSLER, M.D. MCGiLl, F.R.S., PROFESSOR OF MEDICINE, JOHNS HOPKINS UNIVERSITY, BALTIMORE, MARYLAND,

THIs plea is addressed to the resident physicians and the house physicians of the general fever hospitals, and to those practitioners who see a large number of cases of enteric fever. From 7 to 14 per cent. of typhoid fever patients die. During the first 10 years of my service at the Johns Hopkins Hospital there were 63 deaths among 829 patients$7 \cdot 5$ per cent., the minimum rate reached in general hospitals. Of the deaths in typhoid fever 50 per cent. are by astheniaa result of the fevtr, or of the poison, or of both; 25 per cent. from perforation ; from hæmorrhages and other accidents, 25 per cent. Modern methods-hydrotberapy and good nursing - have reduced the death-rate in the asthenic class. The perforation group, as sbown by the statistics of the Brisbane General Hospital (Hare), of the Jobns Hopkins Hospital, and of other institutions, remains much the same as given in the large statistics of Murchison and of Fitz. Whether with early removal of the patients to hospital there may be a still further reduction in the mortality of the asthenic or toxic group, we cannot say; but we can speak positively of a reduction in the death-rate in what bas been the most hopeless of all the complications of the diseaseperforation. I shall not take up space by any discussion of the conditions under which this accident c ccurs. I wish to call attention to the fact that early diagnosis and early operation may save from 30 to 40 per cent. of the cases. To Jan. 1st, 1900, there have been 11 cases operated on from my wards with five recoveries, 45.4 per cent. It is often a desperate remedy for a desperate condition. There are three groups of cases: first, those with so much infiltration and necrosis that the gut is not in a fit condition for operation, and the state of the patient may contra-indicate any attewpt at resection. This was the condition in a recent case. In a second group the operation is successful, but the patient dies in the due course of the disease from the intensity of the fever or in a relapse. In a third group complete recovery takes place. ${ }^{1}$ To incessant watchfulness on the part of my senior assistants and to their constant consulation with the surgical members of the staff $I$ attribute the remarkable percentage of recoveries in our cases.

The classical picture of perforation in typhoid fever is in reality not of perforation but of the consecutive peritonitis, and the vital question is whether we cannot recognise the perforation and hand the patient over to the surgeon within 12 hours of the onset before the peritonitis has become widespread. Unfortunately the symptoms are often obscure, and I know of no more anxious and critical task than that which confronts as in some of these cases. What I am particularly anxious to urge is the careful study of the abdominal symptoms in every case of typhoid fever. Specific instructions should be given to the sister in charge of the ward to notify the house physician at once of any complaint of abdominal pain, of biccough, of vomiting, of sudden rise in pulse or respiration, of unusual sweating, or of signs of collapse. The house physician should bear in mind that perforation is an accident liable to occur in the severe cases, in those with delirium, with diarrhea, or with bæmorrhage, and in those with abdominal symptoms, so that he should be on the watch, and his daily notes should be made with a view of gauging the progress of the symptoms. The following schedule may belp him in drawing up his clinical memoranda.

1. The pain.-(a) Its onset, whether simply an aggravation of the slight abdominal pain such as is common with constipation or with diarrhœea, or whether it is a sudden, intense pain, which causes the patient to call out, and which, though relieved by stupes and ordinary measures, recurs in paroxysms and grows worse. (b) Its locality, whether in

1 It is interesting to note the increasing percentage of recoveries. In Dr. Finney's elaborate analysis of 112 cases in the literature the death-rate was only 21. (Johns Hopkins Hospital Reports, vol, viii.) 
the hypogastric or in the right iliac regions, or in the region of the gall-bladder; whether it is diffuse or localised; whether it radiates towards the pubes or to the penis. It is to be borne in mind that abdominal pain of a severe character in typhoid fever may be associated with an acute pleurisy, with a distended bladder, with a packed rectum, or it may follow an enema, or it may occur with acute cholecystitis.

2. The state of the abdomen.-(a) Whether flat, scaphoid, or distended, and whether, if distended, it is uniform, or chiefly in the hypogastric region. (b) The respiratory movements, whether seen uniformly, and whether below and above the navel. It is to be borne in mind that perforation may be present with a flat or even with a scaphoid abdomen. (c) Palpation. Observe the degree of tension of the abdominal wall, the presence of pain on pressure, the locality of the pain, the extent, the degree of pressure necessary to elicit the pain, and whether it is a pain of increasing severity; the presence or absence of muscle rigidity or spasm, noting particularly its presence or absence in the hy pogastric region and in the right iliac fossa. (d) Percussion. The character of the note in the front of the abdomen and in the flanks. Auscultatory percussion may be helpful when gas is free in the peritoneum. A flat note in the flanks may be present, due to exudate, within 20 hours of the occurrence of the perforation. (e) The liver flatness. Note specifically every third hour after the onset of the symptoms the extent in the middle, nipple, and mid-axillary lines. Remember that obliteration may occur in a flat as well as in a distended abdomen. Complete obliteration in the nipple line may be caused by excessive tympany, but rapid obliteration in a flat, or a not much distended, abdomen is a valuable sign. ( $f$ ) Auscultation. Note the presence or absence of the signs of peristalsis. A friction may be early, within 12 hours of the onset. (g) Examination of the rectum-whether there is tenderness or any fulness between the rectum and the bladder. (h) The stools-the character, frequency, and the presence of blood or sloughs. (i) The urine-whether pain on micturition, frequency, \&c. The onset of symptoms with pain about the bladder or in the penis is not infrequent when perforation takes place in the coils of the ileum in close contact with the bladder.

3. General condition of the patient.-(a) Facies-whether there has been a change in expression; the presence of a risus sardonicus, marked or slight ; the onset of pallor or of sweating; and the signs of collapse, as in cold hands, cold feet, and slight dusky suffusion of the face. (b) The pulse-whether a sudden change in the rate and force; the very rapid, running, thready pulse may not be present for 24 hours after the onset of perforation. (o) The temperature-whether a sudden drop or a sudden rise. (d) Respiration-a sudden increase, which is not infrequent; whether shallow and sighing; $(e)$ Hiccough-sometimes a symptom of onset, more frequently late, when peritonitis is established. ( $f$ ) Vomiting - whether with the onset of the pain it is more frequently a late feature, associated with the presence of the diffuse peritonitis.

4. The blood count. - The constant leukopenia in typhoid fever must be remembered. In a majority of instances in cases well followed there is a rise in the leucocytes, but this is not constant; they may be stationary. Also note the condition of the red blood corpuscles and the hæmoglobin A decided drop may suggest hæmorrhage.

Sudden pain, increasing in intensity, and recurring in paroxysms, is perhaps the most constant symptom of perforation. With an increase of the pulse rate, distension of the abdomen, increasing pain on pressure, and a rise in the leucocytes, the diagnosis is rendered probable. A surgical colleague should be called early, so as to share the responsibility of the case. In a doubtful case the patient should be given the benefit of the doubt and operation should be urged. It is surprising how well a patient, even in a desperate state, may stand it. A general anæsthetic may not be needed. In many cases cocaine has been used, with the help, sometimes, of a whiff of chloroform. One of our cases recovered after three operations in two weeks, two for perforations and one in which a perforation was suspected.

From a surgical standpoint the whole question is reviewed at length by Keen in his "Surgical Complications of Typhoid Fever" and by Dr. Finney and Dr. Cushing in the recently issued Series III. of "Studies in Typhoid Fever," in Johns Hopkins Hospital Reports, vol. viii.

Medicine has become a medico-chirurgical art, and to practise successfully we physicians must know the work of the masters in surgery. In the diagnosis of disorders of the abdomen we are their pupils. In cases likely to become surgical the early association with a surgical colleagne is both a duty and a pleasure.

Baltimore, U.S.A.

\section{OBSERVATIONS BASED ON THE PROBABLE MODE OF FORMATION OF URINARY STONE, RELATIVE TO ITS RECURRENCE AND PREVENTION.}

BY REGINALD HARRISON, F.R.C.S. ENG., SURGEON TO ST. PETER'S HOSPITAL.

IN an article which appeared in THE LANCET ${ }^{1}$ entitled "A Further Contribution to the Surgery of Stone in the Bladder" I endeavoured to illustrate how progressive this work had been during the latter period of the past century and how intimately this was associated with the general adoption of the method of operating which Bigelow originated in 1878 and which has since been known by the name of "litholapaxy." I then purposely left for further consideration the only feature connected with this class of operations which seemed to require amendment or improvement. I refer to the liability to recurrence, which is admittedly larger after crushing as compared with other methods for removing stone from the bladder, though the mortality of the former is considerably less. In the paper referred to there were 101 cases of stone operated upon by litholapaxy alone, in addition to other methods. Of this number there were six deaths, whilst in 23 cases there was recurrence in some degree. The average age of these 23 persons was a fraction under 63 years. Several of the recurrences only amounted to the formation of some phosphatic gravel mixed with tenacious mucus, which was readily removed by the washbottle and aspirator. 17 of these persons had considerable enlargement of the prostate, 12 had pouched bladders, and the same number were more or less dependent upon their catheters at the time of operation. In two cases it seemed probable that recurrence was due to fresh descents of stones from the kidney and their retention within the bladder by the large prostate. In one of the fatal instances recurrence was traceable to a pouched bladder complicated with neglected urethral stricture and chronic suppurative nephritis. Hence it may be inferred that though a stone is successfully removed by operation this does not necessarily imply that the original cause for its production has thus coincidently ceased to exist.

From these statements it seems clear that the class of cases where the tendency to recurrence is greatest and most difficult to deal with, subsequently to the primary operation, is that in which, before stone formed, the bladder was more or less functionally and structurally spoilt by the obstruction it had been submitted to by enlargement of the prostate. It is hardly necessary to enumerate atony or loss of muscular power to expel urine, pouching, trabeculation, and cystitis, as being amongst these commoner effects.

The liabilities in its application to this class of cases seem in some degree to discredit the crushing operation in the eyes of the profession and public. Mr. Oadge in 1886 referred to it in the following terms: "This seems a heavy indictment to bring against lithotrity, but I am afraid there is no gainsaying it, and if so, it would be wrong to pass it over or make light of it. Many of these relapses might be prevented if the patients would observe directions and persevere with treatment." ${ }^{2}$ I shall endeavour to show in detail, later, how we may succeed in giving effect to the latter statement.

On the other hand, in otherwise whealthy persons without these prostatic and other defects the liability to recurrence of calculus in the bladder after operation is slight, as was also shown in the previous paper, and may nsually be accounted for by the unexpected descent of a fresh nucleus from the kidney in the form of a small urate or oxalate

2 Hunterian Lectures, Royal College of Surgeons of England. 